\title{
An Easy Way to Close the Trocar Hole in Obese Patients
}

\author{
Chien-Hua Lin ${ }^{1,2 *}$, Hurng-Sheng $\mathrm{Wu}^{1}{ }^{1}$, Jing-Jim Ou${ }^{1}$, Yueh-Tsung Lee ${ }^{1}$ \\ ${ }^{1}$ IRCAD Taiwan, Department of Surgery, Chang-Bing Show Chwan Memorial Hospital, Taiwan \\ ${ }^{2}$ Department of Surgery, Tri-Service General Hospital, Taiwan \\ Email: "linjh93@yahoo.com.tw
}

Received 16 September 2015; accepted 23 October 2015; published 26 October 2015

Copyright @ 2015 by authors and Scientific Research Publishing Inc.

This work is licensed under the Creative Commons Attribution International License (CC BY).

http://creativecommons.org/licenses/by/4.0/

(c) (i) Open Access

\begin{abstract}
Background: Ten-millimeter ports are often used in laparoscopic surgery. It could be difficult to close the ports sites, especially in obese patients. We described a new method to close ten-millimeter port-site wounds, effective and easily to perform. Methods: Forty cases of laparoscopic bariatric surgery were performed from April 2010 to September 2011 at IRCAD Taiwan. Among them, 30 patients received trocar hole closed with SURGICEL ${ }^{\circledR}$ Original Hemostat and the other 10 patients received trocar hole closed with autologous fat. The results were recorded. Results: In group of SURGICEL ${ }^{\circledR}$ Original Hemostat, includes 21 female and 9 male, age range from 18 to 54 with average 39.5 years. Average BMI is 42.4 (range from 32.6 to 55). The operations include 27 cases of laparoscopic sleeve gastrectomy, 2 cases of laparoscopic gastric bypass, and 1 case of laparoscopic mini-bypass. Group of autologous fat includes 3 female and 7 male, age range from 20 to 49 with average 38.6 years. Average BMI is 39 (range from 32.8 to 64.5). The operations include 9 cases of laparoscopic sleeve gastrectomy, and 1 case of laparoscopic mini-bypass. No complications of trocar wound including infection, hematoma, and hernia were found for a mean follow-up of 6 months. Conclusions: For obese patients, closure of port sites can be difficult. We described a method of closing $10-\mathrm{mm}$ port-sites wounds, which are easy, effective, and not required any special instruments.
\end{abstract}

\section{Keywords}

Bariatric Surgery, Autologous Fat, Troca, Port Site Hernia

\section{Introduction}

Port site hernia is an important yet under-recognized complication of laparoscopic surgery, which carries a high

${ }^{*}$ Corresponding author. 
risk of strangulation due to the small size of the defect involved [1].

The incidence of trocar hernias ranges from $0.2 \%$ to $3.1 \%$ [2]. There are numerous ways of closing laparoscopic wound. However, it is difficult in obese patients [3]. Initially, we used surgicel plug to fill the trocar hole [4]. Then we modified to use autologous fat as a substitute. This idea comes from autologous fat transplantation for breast augmentation. The short outcome is well. We described this easy method.

\section{Materials and Methods}

\subsection{Patients}

Forty cases of laparoscopic bariatric surgery were performed from April 2010 to September 2011 at IRCAD Taiwan. The surgical procedure was performed by the same surgeons, experienced in advanced laparoscopic surgery. None of the patients had undergone previous bariatric surgery.

\subsection{Surgical Technique}

Patients are positioned with legs split in the reverse Trendelenburg position. The surgeon stands between patient's legs. The first $12 \mathrm{~mm}$ trocar is introduced in the midline, 15 to $25 \mathrm{~cm}$ caudal from xyphoid. Three or additional trocars are inserted under direct vision. $12 \mathrm{~mm}$ trocar in left middle abdomen; $5 \mathrm{~mm}$ trocars in right middle and sub-xyphoid process region. Sometimes we will put the fifth trocar is $5 \mathrm{~mm}$ and put in the left lateral abdomen. During operation, we will cut down the fat pad over cardia to identify the angle of His, and the fat pad was prepared for closure of trocar hole. After completion of operation, we will put a close drain the staple line or anastomosis, via $5 \mathrm{~mm}$ trocar wound. The fat pad is moved to the bottom of trocar hole by gasper (Figure 1), then remove the trocar and plug the fat pad in the hole (Figure 2). If the fat pad is not big enough, we will cut down a piece of omentum as a substitute, or the surgical could be also as a substitute (Figure 3).

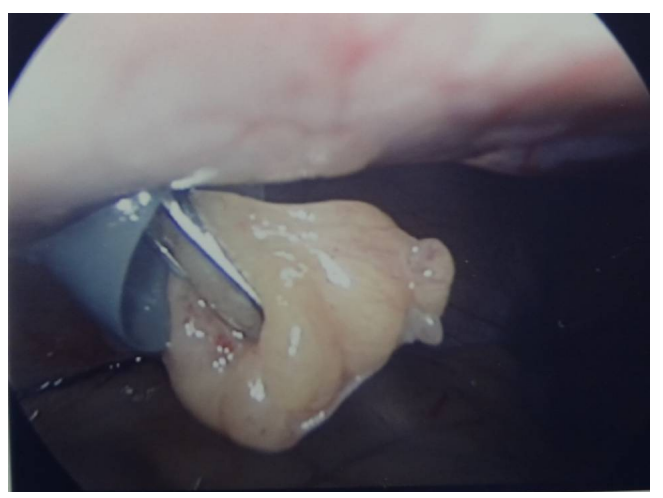

Figure 1. The fat pad is moved to the bottom of trocar hole by gasper.

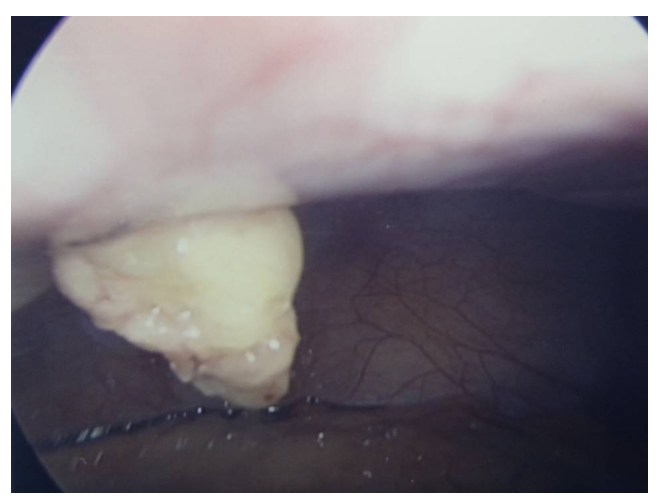

Figure 2. Remove the trocar and plug the fat pad in the hole. 


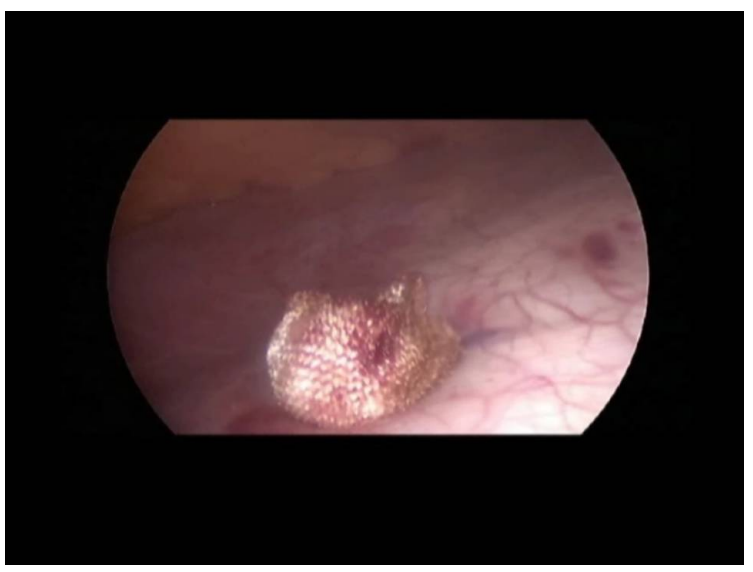

Figure 3. Surgicel could be as a substitute to plug into the hole.

\section{Results}

The demographics of these patients were shown in Table 1.

30 patients received trocar hole closed with SURGICEL ${ }^{\circledR}$ Original Hemostat, includes 21 female and 9 male, age range from 18 to 54 with average 39.5 years. Average BMI is 42.4 (range from 32.6 to 55). The operations include 27 cases of laparoscopic sleeve gastrectomy, 2 case of laparoscopic gastric bypass, and 1 case of laparoscopic mini-bypass.

10 patients received trocar hole closed with autologous fat, includes 3 female and 7 male, age range from 20 to 49 with average 38.6 years. Average BMI is 39 (range from 32.8 to 64.5). The operations include 9 cases of laparoscopic sleeve gastrectomy, and 1 case of laparoscopic mini-bypass.

Post-operative complications were observed in 10\% (4/40) and include leaks (7.5\%), and gastro-gastric fistula (2.5\%). In all patients, no complications of trocar wound including infection, hematoma, and hernia were found for a mean follow-up of 6 months.

Figure 4 and Figure 5 showed the interview of healing of trocar hole. Figure 4 showed 9 months after fat plug in trocar hole, there is no adhesion. Figure 5 showed 15 months after surgical plug in trocar hole, there is no adhesion or incisional hernia was found. However, it showed white color change of peritoneum.

\section{Discussion}

Owens $\mathrm{M}$ et al. described the incidence of port site herniation for bariatric surgery was $0.57 \%$ in 2644 patients with a mean follow-up of 67.4 months [1]. Closure of trocar holes or use of the Versastep trocar system (US Surgical, Norwalk, CT) has the advantage of minimal trocar-related hernias in bariatric surgery [5]. Although post-operative hernia in 5-mm trocar had been reported [6], many authors suggested ten-millimeter ports should be closed [3] [7]. Duron et al. reported that fascia closure of trocar holes might decrease the incidence of hernias and obstructions [8].

There were several methods been reported, included fascia closure device [9], a spinal cord needle [10], a suture carrier [11], a 2-mm trocar [12], or a Deschamps needle [13]. There methods were used to close the fascia and peritoneum together. Chiu CC and Lee WJ et al. presented a new method to close the trocar wound [5]. They use a surgicel plug to put into the trocar hole. However, you need two or three pieces of surgicels. So, we modified our method to use autologous fat as a substitute.

This idea comes from plastic surgeons used autologous fat transplantation for breast augmentation. Autologous fat transplantation was initially performed by Neuber in 1893 to fill in depressed scars [14]. Since then, some plastic surgeons have utilized this method for improving facial and body contour depression and scars. It is commonly used to correct soft tissue defects. Recently, it is popularly used as adjunct in breast reconstruction surgery.

We think surgicel is a foreign body to human body, it may result in inflammation and fibrosis. Brucker $\mathrm{M}$ et al. reported the fate of transplanted autologous fat in animal model [15]. A significant inflammatory response 
Table 1. Characteristics of patients undergoing laparoscopic bariatric surgery for obesity.

\begin{tabular}{ccc}
\hline Variable & Surgicel plug & Fat plug \\
Number & 30 & 10 \\
Age range (average) & $18-54(39.5)$ & $20-49(38.6)$ \\
Gender & 21 & 7 \\
-Female & 9 & 3 \\
-Male & $42.4(32.6-55)$ & $39(32.8-64.5)$ \\
BMI average (range) & & \\
Method & 2 & 1 \\
-LGB & 1 & 9 \\
-Mini-bypass & 27 & 1 \\
-LSG & & 0 \\
Complications & 2 & \\
-Leaks & 1 & \\
-Gastro-gastric fistula & & \\
\hline
\end{tabular}

BMI: body mass index; LGB: laparoscopic gastric bypass; LSG: laparoscopic sleeve gastrectomy.

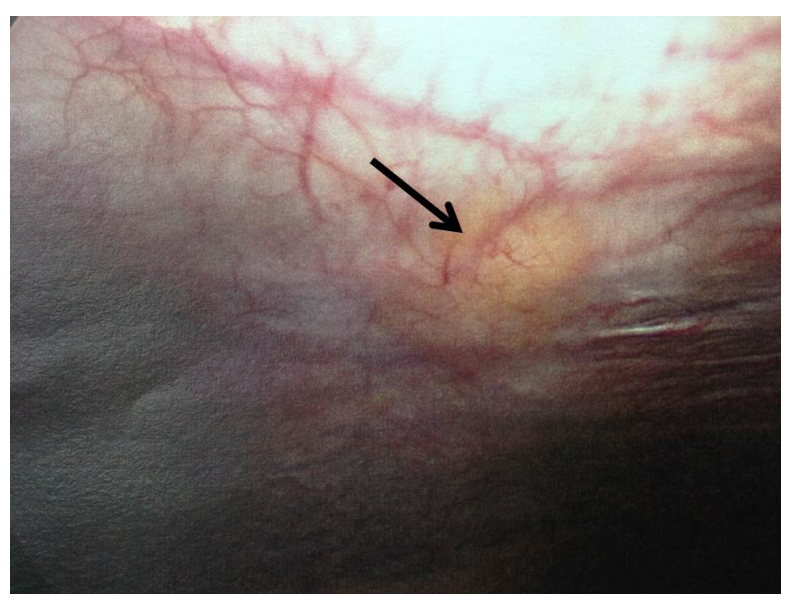

Figure 4. The internal view of fat plug trocar hole 9 months after operation.

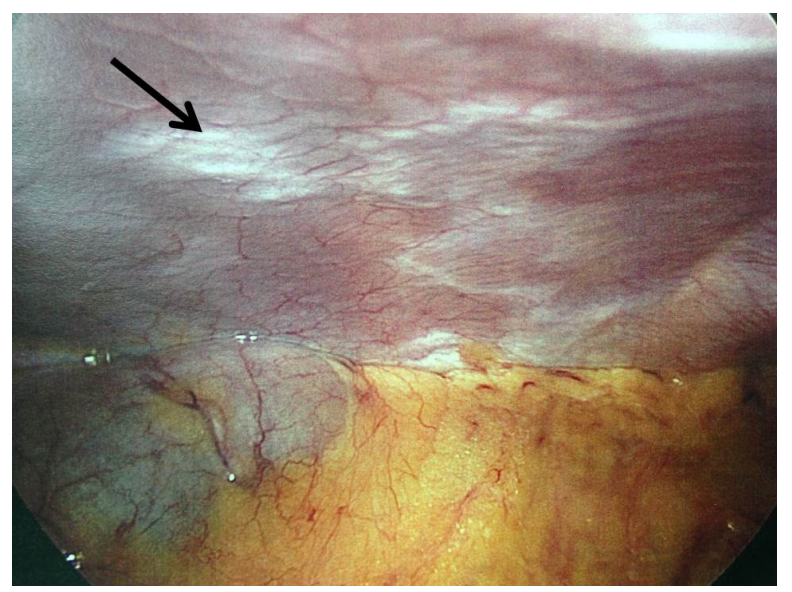

Figure 5. The internal view of mesh plug trocar hole 15 months after operation, white color change of peritoneum. 
was present in the early phases, with evidence of neovascularization. The transplanted fat remained viable at 1 year, with good overall survivability and minimal fibrosis. In this point of view, no matter surgicel or autologous fat, we can achieve the effect of fibrosis in trocar hole.

In our report, we have 10 cases of bariatric surgeries which trocar hole closed with autologous fat, included fat pad of cardia of stomach and greater omentum. There was no port site hernia in the mean follow-up of 6 months. We keep use this method to close the trocar hole and need more cases and longer time to follow up the results.

\section{Conclusion}

In conclusion, autologous fat plug into trocar hole in bariatric surgery is easy, time saving, cost free, and safe.

\section{References}

[1] Owens, M., Barry, M., Janjua, A.Z., et al. (2011) A Systematic Review of Laparoscopic Port Site Hernia in Gastrointestinal Surgery. Surgeon, 9, 218-224. http://dx.doi.org/10.1016/j.surge.2011.01.003

[2] Yamamoto, M., Minikel, L. and Zaritsky, E. (2011) Laparoscopic 5-mm Trocar Site Herniation and Literature Review. JSLS, 15, 122-126.

[3] Durai, R. and Ng, P.C. (2009) Novel Methods of Closing 10-mm Laparoscopic Port-Site Wounds. Journal of Laparoendoscopic \& Advanced Surgical Techniques, 19, 791-793. http://dx.doi.org/10.1089/lap.2009.0023

[4] Chiu, C.C., Lee, W.J., Wang, W., et al. (2006) Prevention of Trocar-Wound Hernia in Laparoscopic Bariatric Operations. Obesity Surgery, 16, 913-918. http://dx.doi.org/10.1381/096089206777822269

[5] Johnson, W.H., Fecher, A.M., McMahon, R.L., et al. (2006) VersaStep Trocar Hernia Rate in Unclosed Fascial Defects in Bariatric Patients. Surgical Endoscopy, 20, 1584-1586. http://dx.doi.org/10.1007/s00464-005-0747-8

[6] Dulskas, A., Lunevičius, R. and Stanaitis, J. (2011) A Case Report of Incisional Hernia through a 5 mm Lateral Port Site Following Laparoscopic Cholecystectomy. Journal of Minimal Access Surgery, 7, 187-189. http://dx.doi.org/10.4103/0972-9941.83512

[7] Hitoshi, T., Yukinari, O., Minako, K., et al. (2004) Trocar Site Hernia. Archives of Surgery, 139, 1248-1256. http://dx.doi.org/10.1001/archsurg.139.11.1248

[8] Duron, J.J., Hay, J.M., Msika, S., et al. (2000) Prevalence and Mechanisms of Small Intestinal Obstruction Following Laparoscopic Abdominal Surgery: A Retrospective Multicenter Study. Archives of Surgery, 135, 208-212. http://dx.doi.org/10.1001/archsurg.135.2.208

[9] Cottam, D.R., Gorecki, P.J., Curvelo, M., et al. (2002) Preperitoneal Herniation into a Laparoscopic Port Site without a Fascial Defect. Obesity Surgery, 12, 121-123. http://dx.doi.org/10.1381/096089202321144702

[10] Petrakis, I., Sciacca, V., Chalkiadakis, G., et al. (1999) A Simple Technique for Trocar Site Closure after Laparoscopic Surgery. Surgical Endoscopy, 13, 1249-1251. http://dx.doi.org/10.1007/PL00011164

[11] Li, P. and Chung, R.S. (1996) Closure of Trocar Wounds Using a Suture Carrier. Surgical Laparoscopy Endoscopy, 6, 469-471. http://dx.doi.org/10.1097/00019509-199612000-00010

[12] Reardon, P.R., McKinney, G. and Craig, E.S. (2003) The 2-mm Trocar: A Safe and Effective Way of Closing Trocar Sites Using Existing Equipment. Journal of the American College of Surgeons, 196, 333-336. http://dx.doi.org/10.1016/S1072-7515(02)01759-3

[13] Di Lorenzo, N., Coscarella, G., Lirosi, F., et al. (2002) Port-Site Closure: A New Problem, an Old Device. JSLS, 6, 181-183.

[14] Pulagam, S.R., Poulton, T. and Mamounas, E.P. (2006) Long-Term Clinical and Radiologic Results with Autologous Fat Transplantation for Breast Augmentation: Case Reports and Review of Literature. Breast Journal, 12, 63-65. http://dx.doi.org/10.1111/j.1075-122X.2006.00188.x

[15] Brucker, M., Sati, S., Spanenberger, A., et al. (2008) Long-Term Fate of Transplanted Autologous Fat in Novel Rabbit Facial Model. Plastic and Reconstructive Surgery, 122, 749-754. http://dx.doi.org/10.1097/PRS.0b013e3181815a41 